\title{
A Simple Bayesian Framework for Content-Based Image Retrieval
}

\author{
Katherine A. Heller \\ Gatsby Computational Neuroscience Unit \\ University College London \\ heller@gatsby $\cdot u c l \cdot a c \cdot u k$
}

\author{
Zoubin Ghahramani* \\ Department of Engineering \\ Cambridge University \\ zoubin@eng.cam.ac.uk
}

\begin{abstract}
We present a Bayesian framework for content-based image retrieval which models the distribution of color and texture features within sets of related images. Given a userspecified text query (e.g. "penguins") the system first extracts a set of images, from a labelled corpus, corresponding to that query. The distribution over features of these images is used to compute a Bayesian score for each image in a large unlabelled corpus. Unlabelled images are then ranked using this score and the top images are returned. Although the Bayesian score is based on computing marginal likelihoods, which integrate over model parameters, in the case of sparse binary data the score reduces to a single matrix-vector multiplication and is therefore extremely efficient to compute. We show that our method works surprisingly well despite its simplicity and the fact that no relevance feedback is used. We compare different choices of features, and evaluate our results using human subjects.
\end{abstract}

\section{Introduction}

As the number and size of image databases grows, accurate and efficient content-based image retrieval (CBIR) systems become increasingly important in business and in the everyday lives of people around the world. Accordingly, there has been a substantial amount of CBIR research, and much recent interest in using probabilistic methods for this purpose (see section 4 for a full discussion). Methods which boost retrieval performance by incorporating user provided relevance feedback have also been of interest.

In this paper we describe a novel framework for performing content-based image retrieval using Bayesian statistics. Even though our method exactly solves a Bayesian inference problem, integrating over model parameters, this reduces to an efficient single matrix-vector multiplication in the presence of sparse binary data. Our method focuses on performing category search, though it could easily be ex-

\footnotetext{
*Also at the Machine Learning Department, Carnegie Mellon University, Pittsburgh, PA, USA
}

tended to other types of searches, and does not require relevance feedback in order to perform reasonably. It also emphasizes the importance of utilizing information given by sets of images, as opposed to single image queries.

In the following sections we describe our Bayesian CBIR system in detail. In section 2 we discuss each component of the system including feature extraction, preprocessing, and the retrieval algorithm. In section 3 we analyze the experimental results from using our system to perform category searches for 50 queries on a Corel image database with nearly 32,000 images. We also analyze texture and color features individually. Lastly, we discuss the large amount of related and future work (sections 4 and 5).

\section{Image Retrieval System}

In our Bayesian CBIR system images are represented as binarized vectors of features. We use color and texture features to represent each image, as described in section 2.1, and then binarize these features across all images in a preprocessing stage, described in section 2.2.

Given a query input by the user, say "penguins", our Bayesian CBIR system finds all images that are annotated "penguins" in a training set. The set of feature vectors which represent these images is then used in a Bayesian retrieval algorithm (section 2.3) to find unlabelled images which portray penguins.

\subsection{Features}

We represent images using two types of texture features, 48 Gabor texture features and 27 Tamura texture features, and 165 color histogram features. We compute coarseness, contrast and directionality Tamura features, as in [1], for each of $9(3 \times 3)$ tiles. We apply 6 scale sensitive and 4 orientation sensitive Gabor filters to each image point and compute the mean and standard deviation of the resulting distribution of filter responses. See [2] for more details on computing these texture features. For the color features we compute an HSV (Hue Saturation Value) 3D histogram [3] such that there are 8 bins for hue and 5 each for value and 
saturation. The lowest value bin is not partitioned into hues since they are not easy for people to distinguish.

\subsection{Preprocessing}

After the 240 dimensional feature vector is computed for each image, the feature vectors for all images in the data set are preprocessed together. The purpose of this preprocessing stage is to binarize the data in an informative way. First the skewness of each feature is calculated across the data set. If a specific feature is positively skewed, the images for which the value of that feature is above the 80th percentile assign the value ' 1 ' to that feature, the rest assign the value ' 0 '. If the feature is negatively skewed, the images for which the value of that feature is below the 20th percentile assign the value ' 1 ', and the rest assign the value '0'. This preprocessing turns the entire image data set into a sparse binary matrix, which focuses on the features which most distinguish each image from the rest of the data set. The one-time cost for this preprocessing is a total of 108.6 seconds for 31,992 images with the 240 features described in the previous section, on a $2 \mathrm{GHz}$ Pentium 4 laptop.

\subsection{Algorithm}

Using the preprocessed sparse binary data, our system takes as input a user-specified text query for category search and outputs images ranked as most likely to belong to the category corresponding to the query. The algorithm our system uses to perform this task is an extension of a recently proposed method for clustering on-demand, called Bayesian Sets [4].

First the algorithm locates all images in the training set with labels that correspond to the query input. Then, using the binary feature vectors which represent the images, the algorithm uses a Bayesian criterion based on marginal likelihoods, to score each unlabelled image as to how well that unlabelled image fits in with the training images corresponding to the query. This Bayesian criterion can be expressed as follows:

$$
\operatorname{score}\left(\mathbf{x}^{*}\right)=\frac{p\left(\mathbf{x}^{*}, \mathcal{D}_{q}\right)}{p\left(\mathbf{x}^{*}\right) p\left(\mathcal{D}_{q}\right)}
$$

where $\mathcal{D}_{q}=\left\{\mathbf{x}_{1}, \ldots \mathbf{x}_{N}\right\}$ are the training images corresponding to the query, and $\mathrm{x}^{*}$ is the unlabelled image that we would like to score. We use the symbol $\mathbf{x}_{i}$ to refer interchangably both to image $i$, and to the binary feature vector which represents image $i$. Each of the three terms in equation 1 are marginal likelihoods and can be written as integrals of the following form:

$$
p\left(\mathbf{x}^{*}\right)=\int p\left(\mathbf{x}^{*} \mid \theta\right) p(\theta) d \theta
$$

Here $\theta$ are the parameters of some distribution which has been chosen to model the image feature vectors, $p(\theta)$ is the prior over these parameters, and $p\left(\mathbf{x}^{*} \mid \theta\right)$ is the likelihood, the probability of observing $\mathrm{x}^{*}$ given that our model is parameterized by $\theta$. Integrating over $\theta$ in equation 2 corresponds to computing the prior probability of observing $\mathrm{x}^{*}$ by averaging over all possible settings of the model parameters. For the query set we have:

$$
p\left(\mathcal{D}_{q}\right)=\int\left[\prod_{i=1}^{N} p\left(\mathbf{x}_{i} \mid \theta\right)\right] p(\theta) d \theta
$$

Here every image $\mathbf{x}_{i}$ in the query set is assumed to be drawn i.i.d. from our model with unknown, but the same parameters $\theta$. Finally, for the numerator of 1 we have:

$$
p\left(\mathbf{x}^{*}, \mathcal{D}_{q}\right)=\int\left[\prod_{i=1}^{N} p\left(\mathbf{x}_{i} \mid \theta\right)\right] p\left(\mathbf{x}^{*} \mid \theta\right) p(\theta) d \theta
$$

Similarly to equation 3 , equation 4 assumes every image in the query set and the image to be scored, $\mathrm{x}^{*}$, all come i.i.d. from our model with unknown, but the same parameters, $\theta$.

Given these marginal likelihoods, we can now intuitively interpret equation 1 as the ratio of the probability that $\mathcal{D}_{q}$ and $\mathrm{x}^{*}$ belong to the same model with the same, though unknown, parameters $\theta$, and the probability that $\mathcal{D}_{q}$ and $\mathbf{x}^{*}$ belong to models with different parameters, $\theta_{1}$ and $\theta_{2}$. The larger this score, the more likely it is that the image we are evaluating, $\mathbf{x}^{*}$, belongs in the same category as the query set of images, $\mathcal{D}_{q}$. Note that this is not the same as computing the point-wise mutual information or testing for independence, since we are comparing models rather than looking at empirical distributions. Moreover, the model in the numerator assumes that the query and the image we are evaluating are dependent through parameters which have been integrated out. After computing this score for every unlabelled image, the highest scoring images are returned to the user.

A general summary of our Bayesian CBIR framework is given in the following psuedocode:

\begin{tabular}{l} 
Bayesian CBIR System \\
\hline background: a set of labelled images $\mathcal{D}_{\ell}$, \\
a set of unlabelled images $\mathcal{D}_{u}$, \\
a probabilistic model $p(\mathbf{x} \mid \theta)$ defined on \\
binary feature vectors representing images, \\
a prior on the model parameters $p(\theta)$ \\
compute texture and color features for each image \\
preprocess: Binarize feature vectors across images \\
input: a text query, $q$ \\
find images corresponding to $q, \mathcal{D}_{q}=\left\{\mathbf{x}_{i}\right\} \subset \mathcal{D}_{\ell}$ \\
for all $\mathbf{x}^{*} \in \mathcal{D}_{u}$ do \\
$\quad$ compute $\quad$ score $\left(\mathbf{x}^{*}\right)=\frac{p\left(\mathbf{x}^{*}, \mathcal{D}_{q}\right)}{p\left(\mathbf{x}^{*}\right) p\left(\mathcal{D}_{q}\right)}$ \\
end for $\quad$ output: sorted list of top scoring images in $\mathcal{D}_{u}$ \\
\hline
\end{tabular}


We still have not described the specific model, $p(\mathbf{x} \mid \theta)$, or addressed the issue of computational efficiency of computing the integrals in 2,3, and 4. Each image $\mathbf{x}_{i} \in \mathcal{D}_{q}$ is represented as a binary vector $\mathbf{x}_{i}=\left(x_{i 1}, \ldots, x_{i J}\right)$ where $x_{i j} \in\{0,1\}$. We define a model in which each element of $\mathbf{x}_{i}$ has an independent Bernoulli distribution:

$$
p\left(\mathbf{x}_{i} \mid \theta\right)=\prod_{j=1}^{J} \theta_{j}^{x_{i j}}\left(1-\theta_{j}\right)^{1-x_{i j}}
$$

The conjugate prior [5] for the parameters of a Bernoulli distribution is the Beta distribution:

$$
p(\theta \mid \alpha, \beta)=\prod_{j=1}^{J} \frac{\Gamma\left(\alpha_{j}+\beta_{j}\right)}{\Gamma\left(\alpha_{j}\right) \Gamma\left(\beta_{j}\right)} \theta_{j}^{\alpha_{j}-1}\left(1-\theta_{j}\right)^{\beta_{j}-1}
$$

where $\alpha$ and $\beta$ are hyperparameters of the prior, and the Gamma function, $\Gamma(\cdot)$ is a generalization of the factorial function. The hyperparameters $\alpha$ and $\beta$ are set empirically from the data, $\alpha=\kappa \mathbf{m}, \beta=\kappa(\mathbf{1}-\mathbf{m})$, where $\mathbf{m}$ is the mean of $\mathbf{x}$ over all images, and $\kappa$ is a scaling factor. For a query $\mathcal{D}_{q}=\left\{\mathbf{x}_{1} \ldots \mathbf{x}_{N}\right\}$ consisting of $N$ vectors it is easy to show that:

$$
p\left(\mathcal{D}_{q} \mid \alpha, \beta\right)=\prod_{j} \frac{\Gamma\left(\alpha_{j}+\beta_{j}\right)}{\Gamma\left(\alpha_{j}\right) \Gamma\left(\beta_{j}\right)} \frac{\Gamma\left(\tilde{\alpha}_{j}\right) \Gamma\left(\tilde{\beta}_{j}\right)}{\Gamma\left(\tilde{\alpha}_{j}+\tilde{\beta}_{j}\right)}
$$

where $\tilde{\alpha}_{j}=\alpha_{j}+\sum_{i=1}^{N} x_{i j}$ and $\tilde{\beta}_{j}=\beta_{j}+N-\sum_{i=1}^{N} x_{i j}$. The other two marginal likelihoods, $p\left(\mathbf{x}^{*}\right)$ and $p\left(\mathbf{x}^{*}, \mathcal{D}_{q}\right)$ from equation 1 can analogously be computed. By combining these three marginal likelihoods in equation 1 , we can compute the score:

$$
\begin{aligned}
\operatorname{score}\left(\mathbf{x}^{*}\right) & =\frac{p\left(\mathbf{x}^{*}, \mathcal{D}_{q}\right)}{p\left(\mathbf{x}^{*}\right) p\left(\mathcal{D}_{q}\right)} \\
=\prod_{j} \frac{\Gamma\left(\alpha_{j}+\beta_{j}+N\right)}{\Gamma\left(\alpha_{j}+\beta_{j}+N+1\right)} \frac{\Gamma\left(\tilde{\alpha}_{j}+x \cdot j\right) \Gamma\left(\tilde{\beta}_{j}+1-x \cdot j\right)}{\Gamma\left(\tilde{\alpha}_{j}\right) \Gamma\left(\tilde{\beta}_{j}\right)} & \frac{\Gamma\left(\alpha_{j}+\beta_{j}\right)}{\Gamma\left(\alpha_{j}+\beta_{j}+1\right)} \frac{\Gamma\left(\alpha_{j}+x \cdot x_{\cdot j}\right) \Gamma\left(\beta_{j}+1-x \cdot j\right)}{\Gamma\left(\alpha_{j}\right) \Gamma\left(\beta_{j}\right)}
\end{aligned}
$$

We can simplify this expression by using the fact that $\Gamma(x)=(x-1) \Gamma(x-1)$ for $x>1$. Also, for each $j$ we can consider the two cases $x_{\cdot j}=0$ and $x_{\cdot j}=1$ separately. For $x_{\cdot j}=1$ we have a contribution $\frac{\alpha_{j}+\beta_{j}}{\alpha_{j}+\beta_{j}+N} \frac{\tilde{\alpha}_{j}}{\alpha_{j}}$. For $x_{\cdot j}=0$ we have a contribution $\frac{\alpha_{j}+\beta_{j}}{\alpha_{j}+\beta_{j}+N} \frac{\tilde{\beta}_{j}}{\beta_{j}}$. Putting these together we can see that:

$$
\operatorname{score}\left(\mathbf{x}^{*}\right)=\prod_{j} \frac{\alpha_{j}+\beta_{j}}{\alpha_{j}+\beta_{j}+N}\left(\frac{\tilde{\alpha}_{j}}{\alpha_{j}}\right)^{x \cdot j}\left(\frac{\tilde{\beta}_{j}}{\beta_{j}}\right)^{1-x \cdot j}
$$

The $\log$ of this score is linear in $\mathbf{x}$ :

$$
\log \operatorname{score}\left(\mathbf{x}^{*}\right)=c+\sum_{j} q_{j} x_{\cdot j}
$$

where

$c=\sum_{j} \log \left(\alpha_{j}+\beta_{j}\right)-\log \left(\alpha_{j}+\beta_{j}+n\right)+\log \tilde{\beta}_{j}-\log \beta_{j}$

and

$$
q_{j}=\log \tilde{\alpha}_{j}-\log \alpha_{j}-\log \tilde{\beta}_{j}+\log \beta_{j}
$$

If we put the entire data set into one large matrix $\mathbf{X}$ with $J$ columns, we can compute the vector $\mathbf{S}$ of $\log$ scores for all images using a single matrix vector multiplication

$$
\mathbf{s}=c+\mathbf{X} \mathbf{q}
$$

For our sparse binary image data, this linear operation can be implemented very efficiently. Each query $\mathcal{D}_{q}$ corresponds to computing vector $\mathbf{q}$ and scalar $c$, which can be done very efficiently as well. The total retrieval time for 31,992 images with 240 features and 1.34 million nonzero elements is 0.1 to 0.15 seconds, on a $2 \mathrm{GHz}$ Pentium 4 laptop.

We can analyze the vector $\mathbf{q}$, which is computed using the query set of images, to see that our algorithm implicitly performs feature selection. We can rewrite equation 11 as follows:

$$
\begin{gathered}
q_{j}=\log \frac{\tilde{\alpha}_{j}}{\alpha_{j}}+\log \frac{\tilde{\beta}_{j}}{\beta_{j}} \\
=\log \left(1+\frac{\sum_{i} x_{i j}}{\alpha_{j}}\right)-\log \left(1+\frac{N-\sum_{i} x_{i j}}{\beta_{j}}\right)
\end{gathered}
$$

If the data is sparse and $\alpha_{j}$ and $\beta_{j}$ are proportional to the data mean number of ones and zeros respectively, then the first term dominates, and feature $j$ gets weight approximately:

$$
q_{j} \approx \log \left(1+\text { const } \frac{\text { querymean }_{j}}{\text { datamean }_{j}}\right)
$$

when that feature appears in the query set, and a relatively small negative weight otherwise. A feature which is frequent in the query set but infrequent in the overall data will have high weight. So, a new image which has a feature (value 1) which is frequent in the query set will typically receive a higher score, but having a feature which is infrequent (or not present in) the query set lowers its score.

\section{Results}

We used our Bayesian CBIR system to retrieve images from a Corel data set of 31,992 images. 10,000 of these images were used with their labels as a training set, $\mathcal{D}_{\ell}$, while the rest comprised the unlabelled test set, $\mathcal{D}_{u}$. We tried a total of 50 different queries, corresponding to 50 category searches, and returned the top 9 images retrieved for each query using both texture and color features, texture features alone, and color features alone. We used the given labels 
for the images in order to select the query set, $\mathcal{D}_{q}$, out of the training set. To evaluate the quality of the labelling in the training data we also returned a random sample of 9 training images from this query set. In all of our experiments we set $\kappa=2$.

The above process resulted in 1800 images: 50 queries $\times 9$ images $\times 4$ sets (all features, texture features only, color features only, and sample training images). Two uninformed human subjects were then asked to label each of these 1800 images as to whether they thought each image matched the given query. We chose to compute precisions for the top nine images for each query based on factors such as ease of displaying the images, reasonable quantities for human hand labelling, and because when people perform category searches they generally care most about the first few results that are returned. We found the evaluation labellings provided by the two human subjects to be highly correlated, having correlation coefficient 0.94 .

We then compared our Bayesian CBIR results on all features with the results from using two different nearest neighbor algorithms to retrieve images given the same image data set, query sets and features. The first nearest neighbor algorithm found the nine images which were closest (euclidean distance) to any individual member of the query set. This algorithm is approximately 200 times slower than our Bayesian approach. More analagous to our algorithm, the second nearest neighbor algorithm found the nine images which were closest to the mean of the query set. Lastly we compared to the Behold Image Search online [17]. Behold Image Search online runs on a more difficult 1.2 million image dataset. We compare to the Behold system because it is a currently available online CBIR system which is fast and handles query words, and also because is was part of the inspiration for our own Bayesian CBIR system. The results given by these three algorithms were similarly evaluated by human subjects.

The results from these experiments are given in table 1. The first column gives the query being searched for, the second column is the number of images out of the nine images returned by our algorithm which were labelled by the human subjects as being relevant to the query (precision $\times 9$ ). The third and fourth columns give the same kind of score for our system, but restricting the features used to texture only and color only, respectively. The fifth column shows the results of the Behold online system, where N/A entries correspond to queries which were not in the Behold vocabulary. The sixth and seventh columns give the results for the nearest neighbor algorithms using all members of the query set and the mean of the query set respectively. The eighth column gives the number of images out of the 9 randomly displayed training images that were labelled by our subjects as being relevant to the query. This gives an indication of the quality of the labelling in the Corel data. The last col- umn shows the number of training images, $n$, which comprise the query set (i.e. they were labelled with the query word in the labellings which come with the Corel data).

Looking at the table we can notice that our algorithm using all features (BIR) performs better than either the texture features (BIRtex) or color features alone (BIRcol); the pvalues for a Binomial test for texture or color features alone performing better than all features are less than 0.0001 in both cases. In fact our algorithm can do reasonably well even when there are no correct retrievals using either color or texture features alone (see, for example, the query "eiffel"). Our algorithm also substantially outperforms all three of the comparison algorithms (BO, NNmean, NNall). It tends to perform better on examples where there is more training data, although it does not always need a large amount of training data to get good retrieval results; in part this may result from the particular features we are using. Also, there are queries (for example, "desert") for which the results of our algorithm are judged by our two human subjects to be better than a selection of the images it is training on. This suggests both that the original labels for these images could be improved, and that our algorithm is quite robust to outliers and poor image examples. Lastly, our algorithm finds at least 1 , and generally many more, appropriate images, in the nine retrieved images, on all of the 50 queries.

The average number of images returned, across all 50 queries, which were labelled by our subjects as belonging to that query category, are given in figure 1 . The error bars show the standard error about the mean. Some sample images retrieved by our algorithm are shown in figures 4-7, where the queries are specified in the figure captions.

By looking at these examples we can see where the algorithm performs well, and what the algorithm mistakenly assigns to a particular query when it does not do well. For example, when looking for "building" the algorithm occasionally finds a large vertical outdoor structure which is not a building. This gives us a sense of what features the algorithm is paying attention to, and how we might be able to improve performance through better features, more training examples, and better labelling of the training data. We also find that images which are prototypical of a particular query category tend to get high scores (for example, the query "sign" returns very prototypical sign images).

We also compute precision-recall curves for our algorithm and both nearest neighbor variants that we compared to (figure 2). For the precision-recall curves we use the labellings which come with the Corel data. Both nearest neighbor algorithms perform significantly worse than our method. NNall has a higher precision than our algorithm at the lowest level of recall. This is because there is often at least one image in the Corel test set which is basically identical to one of the training images (a common criticism 


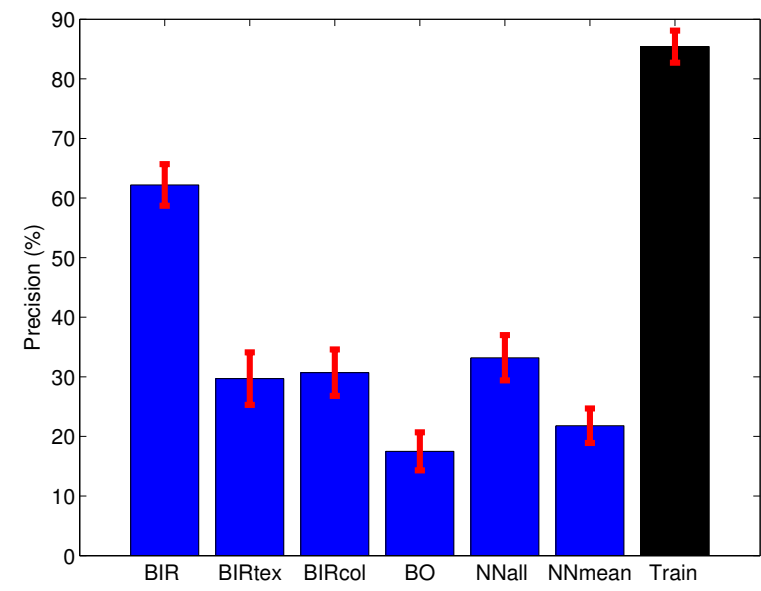

Figure 1 . mean \pm s.e. $\%$ correct retrievals over 50 queries

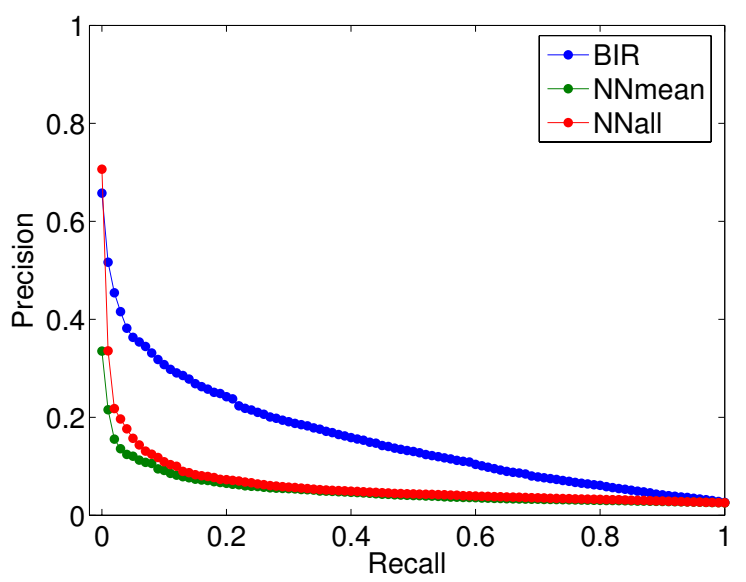

Figure 2. Precision-recall curves for our method (blue) and both nearest neighbor comparison methods, averaged over all 50 queries, and using the Corel data labellings

of this particular data set). The precision of NNall immediately falls because there are few identical images for any one query, and generalization is poor. Our algorithm does not preferentially return these identical images (nor does NNmean), and they are usually not present in the top 9 retrieved.

Four sets of retrieved images (all features, texture only, color only, and training) for all 50 queries can be found in additional materials ${ }^{1}$, which we encourage the reader to have a look through.

\section{Related Work}

There is a great deal of literature on content-based image retrieval. An oft cited early system developed by IBM was "Query by Image Content" (QBIC [6]). A thorough review

\footnotetext{
1http://www.gatsby $\cdot$ ucl.ac.uk/ heller/BIRadd.pdf
}

of the state of the art until 2000 can be found in [7].

We limit our discussion of related work to (1) CBIR methods that make use of an explicitly probabilistic or Bayesian approach, (2) CBIR methods that use sets of images in the context of relevance feedback, and (3) CBIR methods that are based on queries consisting of sets of images.

Vasconcelos and Lippman have a significant body of work developing a probabilistic approach to content-based image retrieval (e.g. [8]). They approach the problem from the framework of classification, and use a probabilistic model of the features in each class to find the maximum a posteriori class label. In [9] the feature distribution in each class is modelled using a Gaussian mixture projected down to a low dimensional space to avoid dimensionality problems. The model parameters are fit using EM for maximum likelihood estimation. Our approach differs in several respects. Firstly, we employ a fully Bayesian approach which involves treating parameters as unknown and marginalizing them out. Second, we use a simpler binarized feature model where this integral is analytic and no iterative fitting is required. Moreover, we represent each image by a single feature vector, rather than a set of query vectors. Finally, we solve a different problem in that our system starts with a text query and retrieves images from an unlabelled data set- the fact that the training images are given a large number of non-mutually exclusive annotations suggests that the classification paradigm is not appropriate for our problem.

PicHunter [10] is a Bayesian approach for handling relevance feedback in content based image retrieval. It models the uncertainty in the users' goal as a probability distribution over goals and uses this to optimally select the next set of images for presentation.

PicHunter uses a weighted pairwise distance measure to model the similarity between images, with weights chosen by maximum likelihood. This is quite different from our approach which models the joint distribution of sets of images averaging over model parameters.

Rui et al [11] explore using the $\mathrm{tf}^{-i d f^{2}}$ representation from document information retrieval in the context of image retrieval. They combine this representation with a relevance feedback method which reweights the terms based on the feedback and report results on a dataset of textures. It is possible to relate tf-idf to the feature weightings obtained from probablistic models but this relation is not strong.

Yavlinsky et al [12] describe a system for both retrieval and annotation of images. This system is based on modeling $p(\mathbf{x} \mid w)$ where $\mathbf{x}$ are image features and $w$ is some word from the annotation vocabulary. This density is modeled using a non-parameteric kernel density estimator, where the kernel uses the Earth Mover's Distance (EMD). Bayes rule is used to get $p(w \mid \mathbf{x})$ for annotation.

\footnotetext{
${ }^{2}$ term-frequency inverse-document-frequency
} 

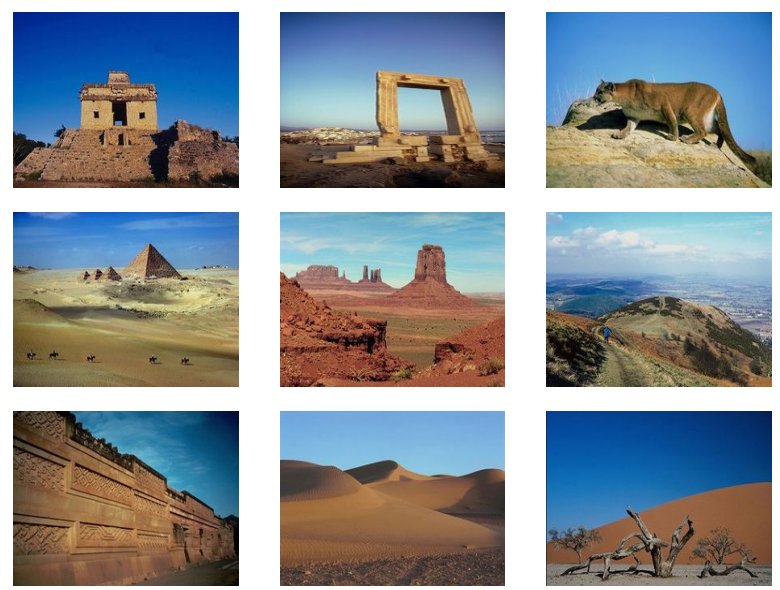

Figure 3. Query: desert
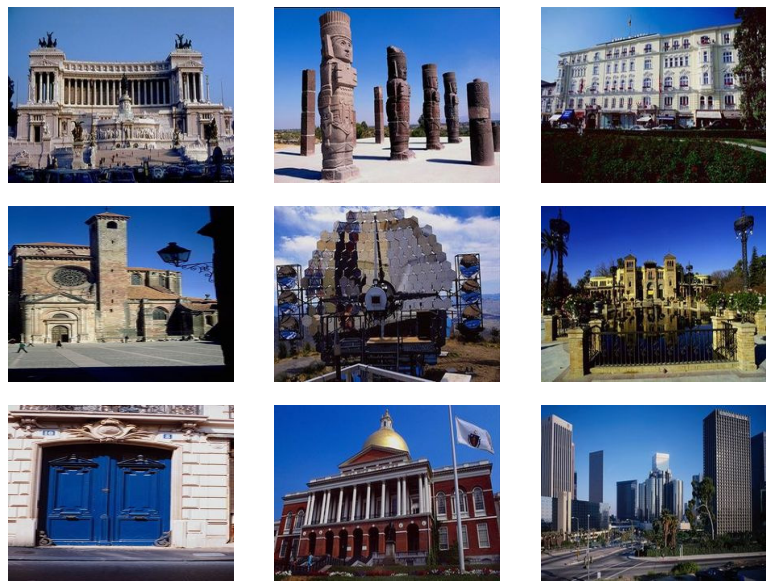

Figure 4. Query: building
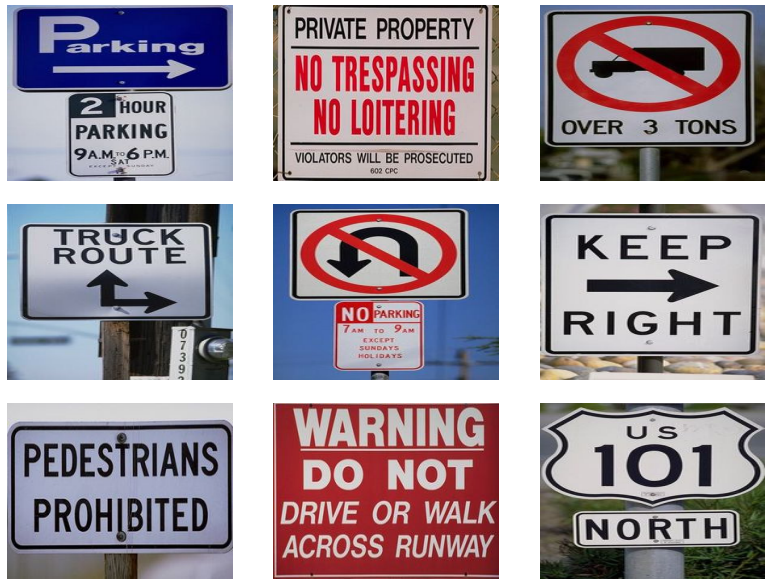

Figure 5. Query: sign

Gosselin and Cord [13] investigate active learning approaches to efficient relevance feedback using binary classifiers to distinguish relevant and irrelevant classes. Among
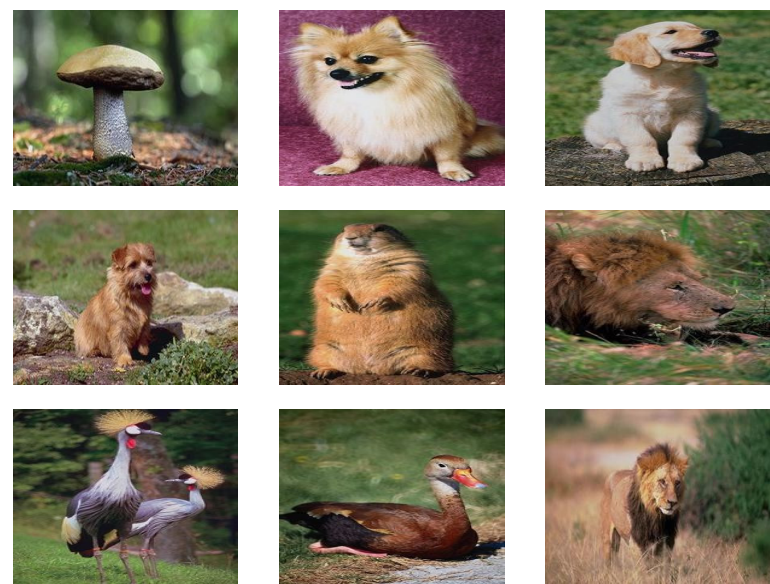

Figure 6. Query: pet
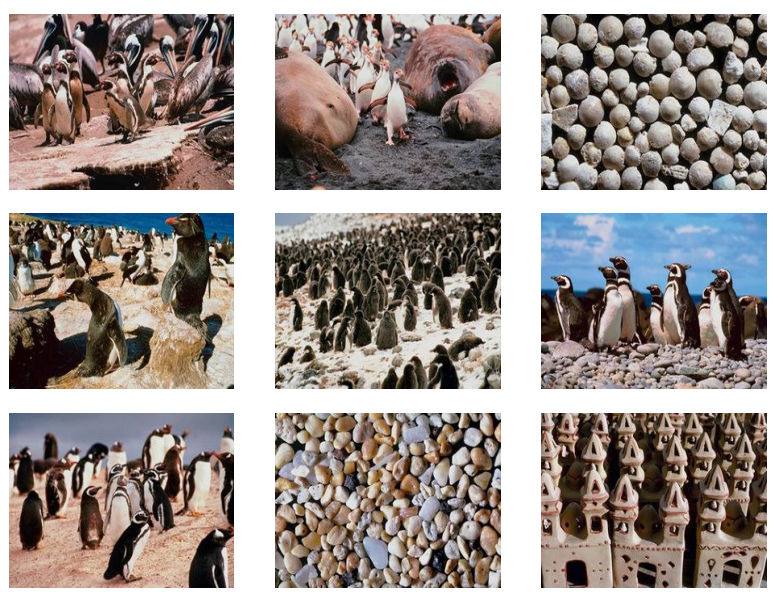

Figure 7. Query: penguins

other methods, they compare a "Bayes classifier" which uses Parzen density estimators with a fixed-width Gaussian kernel to model $P(\mathbf{x} \mid$ relevant $)$ and $P(\mathbf{x} \mid$ irrelevant $)$ where $\mathbf{x}$ are image features. Our approach differs in several respects. First, we model the probablity of the target $\mathbf{x}$ belonging to a cluster while integrating out all parameters of the cluster, and compare this to the prior $p(\mathbf{x})$. Strictly speaking, Parzen density estimators are not Bayesian in that they do not define a prior model of the data, but rather can be thought of as frequentist smoothers for the empirical distribution of the data. They thereby lose important properties of Bayesian methods-namely the ability to compute marginal likelihoods, which are key to our method.

The combination of labeled and unlabeled data and the sequential nature of relevance feedback, mean that active learning approaches are very natural for CBIR systems. Hoi and Lyu [14] adapt the semi-supervised active learning framework of Zhu et al [15] as a way of incorporating 
relevance feedback in image retrieval.

In [16], the user manually specifies a query consisting of a set of positive and negative example images. The system then finds images which minimize the distance in color histogram space to the positive examples, while maximizing distance to the negative examples. While our method is not directly based on querying by examples, since it uses text input to extract images from a labelled set, it implicitly also uses a set of images as the query. However, in our system the set only contains positive examples, the user only has to type in some text to index this set, and the subsequent retrieval is based on different principles.

\section{Conclusions and Future Work}

We have described a new Bayesian framework for content-based image retrieval. We show the advantages of using a set of images to perform retrieval instead of a single image or plain text. We obtain good results from using a Bayesian criterion, based on marginal likelihoods, to find images most likely to belong to a query category. We also show that this criterion can be easily and efficiently computed as a matrix-vector multiplication when image feature vectors are sparse and binary.

In all of our experiments, the two free parameters, the preprocessing percentile threshold for binarizing the feature vectors and $\kappa$, the scaling factor for setting the hyperparameters, are set to 20 and 2 respectively. In our experience, this initial choice of values seemed to work well, but it would be interesting to see how performance varies as we adjust the values of these two parameters.

In the future there are many extensions which we would like to explore. We plan to extend the system to incorporate multiple word queries where the query sets from all words in the query are combined by either taking the union or the intersection. We would also like to look into incorporating relevance feedback, developing revised query sets, in our Bayesian CBIR system. By combining with relevance feedback, the principles used here can also be applied to other types of seaches, such as searching for a specific target image. Lastly, we would like to explore using our Bayesian CBIR framework to perform automatic image annotation as well as retrieval.

Acknowledgements: Thanks to Alexei Yavlinsky for help with the dataset and feature extraction. ZG was supported partly by the DARPA CALO project at CMU.

\section{References}

[1] H. Tamura, S. Mori, and T. Yamawaki. Textual features corresponding to visual perception. IEEE Trans on Systems, Man and Cybernetics, 8:460-472, 1978.
[2] P. Howarth and S. Rüger. Evaluation of texture features for content-based image retrieval. In International Conference on Image and Video Retrieval (CIVR), 2004.

[3] D. Heesch, M. Pickering, S. Rüger, and A. Yavclinsky. Video retrieval with a browsing framework using key frames. In Proceedings of TRECVID, 2003.

[4] Z. Ghahramani and K. A. Heller. Bayesian sets. In Advances in Neural Information Processing Systems, volume 18, 2005.

[5] J. Bernardo and A. Smith. Bayesian Theory. Wiley, 2000.

[6] M. Flickner, H. Sawhney, W. Niblack, J. Ashley, Q. Huang, B. Dom, M. Gorkani, J. Hafner, D. Lee, D. Petkovic, D. Steele, and P.Yanker. Query by image and video content: the qbic system. IEEE Computer, 28:23-32, 1995.

[7] A. W. M. Smeulders, M. Worring, S. Santini, A. Gupta, and R. Jain. Content-based image retrieval at the end of the early years. IEEE Transactions on Pattern Analysis and Machine Intelligence, 22(12), 2000.

[8] N. Vasconcelos and A. Lippman. A bayesian framework for content-based indexing and retrieval. In Proceedings of IEEE Data Compression Conference, 1998.

[9] N. Vasconcelos. Minimum probability of error image retrieval. IEEE Transactions on Signal Processing, 52(8), 2004.

[10] I. Cox, M. Miller, T. Minka, T. Papathornas, and P. Yianilos. The bayesian image retrieval system, pichunter: Theory, implementation, and psychophysical experiments. IEEE Tran. On Image Processing, 9:20-37, 2000.

[11] Y. Rui, T. Huang, and S. Mehrotra. Content-Based image retrieval with relevance feedback in MARS. In Proceedings of IEEE International Conference on Image Processing, pages 815-818, 1997.

[12] A Yavlinsky, E Schofield, and S Rüger. Automated image annotation using global features and robust nonparametric density estimation. In Proceedings of the International Conference on Image and Video Retrieval, 2005.

[13] P. H. Gosselin and M. Cord. A comparison of active classification methods for content-based image retrieval. In First International Workshop on Computer Vision meets Databases (CVDB 2004), 2004.

[14] S. C. H. Hoi and M. R. Lyu. A semi-supervised active learning framework for image retrieval. In Proceedings of IEEE Computer Society Conference on Computer Vision and Pattern Recognition (CVPR 2005), 2005.

[15] X. Zhu, J. Lafferty, and Z. Ghahramani. Combining active learning and semi-supervised learning using gaussian fields and harmonic functions. In Proceedings of the ICML2003 Workshop on the Continuum from Labeled to Unlabeled Data, 2003.

[16] J. Assfalg, A. Del Bimbo, and P. Pala. Using multiple examples for content-based image retrieval. In Proceedings of the IEEE International Conference on Multimedia and Expo (ICME 2000), 2000.

[17] Behold Image Search. Multimedia and Information Systems Group, Imperial College London. http://grouse.doc.ic.ac.uk:8800/searchvis.jsp 


\begin{tabular}{|c|c|c|c|c|c|c|c|c|}
\hline Query & BIR & BIRtex & BIRcol & $\mathrm{BO}$ & NNmean & NNall & Train & \# Train \\
\hline abstract & 8 & 4 & 8 & 5.5 & 2 & 1 & 5 & 391 \\
\hline aerial & 4 & 0.5 & 2 & 0 & 2 & 3 & 8 & 201 \\
\hline animal & 8 & 5 & 6 & 1 & 3 & 9 & 9 & 1097 \\
\hline ape & 4 & 1 & 0 & 0 & 2 & 7 & 8.5 & 27 \\
\hline boat & 1 & 0 & 1 & 0.5 & 1 & 1 & 7 & 61 \\
\hline building & 7.5 & 9 & 2.5 & 4 & 6 & 5.5 & 8 & 1207 \\
\hline butterfly & 5 & 4 & 1 & 1 & 2 & 0 & 9 & 112 \\
\hline castle & 3.5 & 2 & 2 & 1 & 0 & 3 & 8 & 229 \\
\hline cavern & 5.5 & 1 & 2.5 & 0.5 & 2 & 1 & 9 & 34 \\
\hline cell & 6 & 0 & 5 & 9 & 5 & 4 & 8 & 29 \\
\hline church & 3.5 & 1 & 2 & 0 & 5 & 0 & 6 & 173 \\
\hline clouds & 5 & 5.5 & 1.5 & 0 & 3 & 5 & 5.5 & 604 \\
\hline coast & 7 & 3 & 2 & 1 & 2 & 2 & 9 & 299 \\
\hline desert & 4.5 & 0 & 1 & 1 & 0 & 1.5 & 2 & 168 \\
\hline door & 8.5 & 8 & 1 & 0 & 2 & 0 & 5.5 & 92 \\
\hline drawing & 4 & 0 & 0 & 2 & 7 & 3 & 9 & 69 \\
\hline eiffel & 6 & 0 & 0 & N/A & 0 & 0 & 8.5 & 15 \\
\hline fireworks & 9 & 9 & 3 & 0 & 1 & 3 & 9 & 76 \\
\hline flower & 9 & 1 & 7.5 & 2 & 3 & 1 & 9 & 331 \\
\hline fractal & 3 & 0 & 5.5 & 0.5 & 0 & 2 & 8.5 & 43 \\
\hline fruit & 5.5 & 0.5 & 6.5 & 0 & 0 & 1 & 8 & 199 \\
\hline house & 6 & 8 & 0 & 1.5 & 1 & 2 & 8 & 184 \\
\hline kitchen & 6 & 1 & 2 & N/A & 5 & 3 & 9 & 32 \\
\hline lights & 6.5 & 3 & 1.5 & N/A & 1 & 0 & 7 & 203 \\
\hline model & 5 & 4 & 0 & N/A & 3 & 4 & 9 & 102 \\
\hline mountain & 6 & 1 & 2.5 & 1 & 2 & 3 & 8 & 280 \\
\hline mountains & 7 & 2 & 8 & N/A & 1 & 3 & 8.5 & 368 \\
\hline penguins & 6 & 1 & 5 & N/A & 0 & 0 & 8.5 & 34 \\
\hline people & 6 & 2 & 0 & 1.5 & 4 & 5 & 8.5 & 239 \\
\hline person & 4 & 0.5 & 1.5 & 1.5 & 4 & 5 & 7.5 & 114 \\
\hline pet & 3 & 2 & 2 & 0.5 & 0 & 4.5 & 8.5 & 138 \\
\hline reptile & 3 & 1 & 1 & 1 & 0 & 1 & 9 & 99 \\
\hline river & 4.5 & 1.5 & 4.5 & 1.5 & 2 & 4 & 7 & 211 \\
\hline sea & 7.5 & 6 & 3 & 0.5 & 2 & 3 & 6 & 90 \\
\hline sign & 9 & 9 & 1 & 8 & 1 & 0 & 9 & 53 \\
\hline snow & 6 & 0 & 4 & 1 & 2 & 3 & 9 & 259 \\
\hline stairs & 3 & 3.5 & 2 & 0 & 1 & 2 & 8 & 53 \\
\hline sunset & 9 & 7.5 & 4 & 2.5 & 3 & 2.5 & 8.5 & 187 \\
\hline textures & 7 & 8 & 1 & N/A & 0 & 8 & 3 & 615 \\
\hline tool & 4 & 1 & 4 & 1 & 1 & 5 & 9 & 28 \\
\hline tower & 7.5 & 3.5 & 0.5 & 2.5 & 3 & 2.5 & 6 & 138 \\
\hline trees & 9 & 1 & 8 & N/A & 6 & 8 & 8.5 & 1225 \\
\hline turtle & 2 & 0 & 1 & N/A & 0 & 0 & 9 & 13 \\
\hline urban & 7.5 & 4.5 & 2 & N/A & 3 & 3 & 9 & 133 \\
\hline volcano & 2 & 0 & 3 & 0 & 0 & 0 & 3 & 54 \\
\hline water & 9 & 3 & 5.5 & 0 & 5 & 9 & 5.5 & 1863 \\
\hline waterfall & 2 & 0 & 2 & 1 & 0 & 3 & 9 & 103 \\
\hline white & 9 & 3 & 9 & 4.5 & 1 & 6.5 & 7.5 & 240 \\
\hline woman & 4 & 2 & 0 & 3 & 2 & 3 & 8.5 & 181 \\
\hline zebra & 2 & 0 & 0 & N/A & 0 & 2 & 8 & 21 \\
\hline
\end{tabular}

Table 1. Results table over 50 queries 